\title{
Research Paper \\ The Relationship Between Spiritual Well-Being and Care Burden in Unofficial Care- givers of Elderly Patients With Dementia
}

\author{
Mahdiyeh Salehi ${ }^{1}$ (D), Masoud Ahmadzad Asl ${ }^{2}$, Ruohollah Seddigh ${ }^{3}$, Vahid Rashedi ${ }^{1}$, Mostafa Almasi-Dooghaee ${ }^{4}$, Leila \\ Kamalzadeh ${ }^{5}$ * Behnam Shariati ${ }^{5}$ (1)
}

\author{
1. School of Behavioral Science and Mental Health (Tehran Institute of Psychiatry), Iran University of Medical Science, Tehran, Iran. \\ 2. Research Center of Addiction and Risky Behavior, Iran University of Medical Sciences, Tehran, Iran. \\ 3. Spiritual Health Research Center, Iran University of Medical Sciences, Tehran, Iran. \\ 4. Firoozgar Medical Education, Iran University of Medical Sciences, Tehran, Iran. \\ 5. Mental Health Research Center, Iran University of Medical Sciences, Tehran, Iran.
}

\begin{tabular}{|c|c|}
\hline $\begin{array}{l}\text { Use your device to scan } \\
\text { and read the article online }\end{array}$ & ditation Salehi M, Ahmadzad Asl M, Seddigh R, Rashedi V, Almasi-Dooghaee M, Kamalzadeh L, Shariati B. [The Rela- \\
\hline 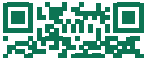 & tionship Between Spiritual Well-Being and Care Burden in Unofficial Caregivers of Elderly Patients With Dementia (Persian)]. \\
\hline 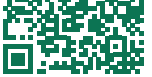 & dol'http://dx.doi.org/10.32598/ijpcp.26.1.2658.1 \\
\hline
\end{tabular}

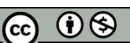

Received: 29 May 2019

Accepted: 26 Jan 2020

Available Online: 01 Apr 2020

Key words:

Care burden, Dementia, Spirituality, Elderly

\section{A B STRACT}

Objectives Dementia is a common disease in the elderly and imposes a heavy care burden on family members. Many factors can be associated with care burden. One of these factors can be the caregivers' Spiritual Well-Being (SWB). This study aimed to examine the relationship between SWB and care burden in caregivers of dementia patients.

Methods This correlational study was conducted on 60 caregivers ( $75 \%$ female and $25 \%$ male with a mean age of 47 years) of patients with dementia referred to Rasoul-e-Akram Hospital and Brain \& Cognitive Clinics in Tehran, Iran in 2019. Samples were selected using purposive and convenience sampling methods. The caregivers were evaluated by using the Caregiver Burden Questionnaire and SWB Scale and a socio-demographic checklist. Data were analyzed by using Pearson's correlation coefficient and multiple linear regression analysis in SPSS software at the significance level of $\mathrm{P}<0.05$.

Results About $16.7 \%$ of caregivers had little care burden, 35\% had moderate to severe burden, $25 \%$ moderate burden and $23.3 \%$ severe burden. Moreover, $1.7 \%$ had poor SWB, 66.7\% moderate SWB and $31.7 \%$ high SWB. The care burden had a negative and non-significant relationship with religious dimension of SWB ( $r=-0.089, P=0.505)$, and a negative and significant relationship with existential dimension of SWB ( $r=-0.283, P=0.032)$. Linear regression model revealed that existential dimension had a relationship with care burden $(\beta=-0.298, P=0.023)$ and explained $9 \%$ of variation in care burden.

Conclusion SWB plays an important role in improving the overall health of caregivers. in addition to being one of determinant of care burden, it acts as a factor in enhancing other aspects of health. Overall, caregiving is a stressful job; being aware of positive topics such as spirituality helps therapists provide strategies for caregivers to reduce their stress and care burden.

\section{* Corresponding Author: \\ Behnam Shariati, MD.}

Address: Mental Health Research Center, Iran University of Medical Sciences, Tehran, Iran.

Tel: +98 (21) 66506862

E-mail: behnamshariatimd@gmail.com 


\section{Extended Abstract}

\section{Introduction}

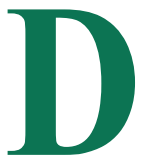

ementia is a chronic illness with different signs and symptoms such as memory impairment, language impairment, psychological changes, and behavioral disorders [1]. Cognitive impairment, increased behavioral and emotional problems and daily living activities impose a considerable care burden on individuals and society [2]. This disease is a global challenge that directly affects 47.5 million people worldwide and affects 7.7 million people each year. In most parts of the world, the prevalence of dementia in people over 60 years is $5-7 \%$ [3]. In Iran, a study in five provinces reported the prevalence of dementia as $7.7 \%$ [4]. Its prevalence is also high in old patients admitted to hospitals, about 12.9-63\% [5, 6]. Given the high prevalence of this disease among the elderly, the different needs of these patients as well as its high medical costs and care burden is a challenge to health care systems $[1,7]$. Family and spouses of the elderly people play an important role in improving the care of patients with dementia. Most of these caregivers play their role full time and give themselves little time. Sleep disorders, the caregiver's poor physical health, low life satisfaction, and high depression and anxiety are some of the consequences that result from high care burden for these individuals $[2,8]$.

Systematic reviews of the care burden among people with dementia have shown that the behavioral problems and psychological symptoms of dementia are the most important factors related to the care burden. Demographic and psychological factors of the caregiver are also another factors affecting care burden [9]. Spiritual Well-Being (SWB) is one of the psychological factors that can play an important role in reducing the care burden of caregivers. According the World Health Organization, health is a concept with physical, social, psychological and spiritual dimensions [10]. Among these dimensions, SWB is a concept that is less understood and is more challenging. Religious beliefs act as a shield against stress, depression, delinquency, and other health-damaging factors [11]. Numerous studies have been conducted on the relationship between SWB as a protective factor in reducing the care burden for various diseases $[12,13]$. The purpose of this study was to investigate the relationship between SWB and care burden in elderly people with dementia.

\section{Method}

This correlational study was conducted on 60 caregivers of dementia patients referred to Rasoul-e-Akram Hospital and brain and cognition clinics in Tehran, Iran in 2018. Samples were selected by purposive sampling method. The inclusion criteria for the caregivers were: caring for patients with diagnosed disease by specialists, caregiving for at least 20 hours per week, being a relative of the patient, and having at least elementary primary education to complete the questionnaire. In addition to a form for recording demographic and contextual characteristics of patients (age, gender, education, marital status, financial status, length of caregiving, disease duration), two valid and reliable questionnaires were used including Caregiver Burden Questionnaire and SWB Scale.

\section{Results}

About $16.7 \%$ of caregivers had little care burden, $35 \%$ moderate to severe burden, $25 \%$ moderate burden and $23.3 \%$ severe burden. Moreover, $1.7 \%$ of caregivers had poor SWB, $66.7 \%$ moderate SWB and $31.7 \%$ high SWB. According to the results of multiple linear regression analysis, among the entered variables (some demographic factors as covariate variables and two SWB dimensions of religious and existential well-beings), only the existential well-being remained in the model $(\beta=-0.298, \mathrm{P}=0.023)$ which accounted for about $9 \%$ of changes in care burden (Table 1).

Table 1. Distribution of spiritual health variables in caregivers

\begin{tabular}{cc}
\hline Total Score/ Dimension & Mean (Standard deviation) \\
\hline Existential Dimension & $(52 / 10) 25 / 47$ \\
\hline Religious Dimension & $(33 / 10) 18 / 43$ \\
\hline Spiritual health (Total Score) & $(05 / 19) 53 / 90$ \\
\hline Total Caregiver's burden Score & $(62 / 21) 11 / 46$ \\
\hline
\end{tabular}




\section{Discussion}

The present study showed a significant relationship between the SWB dimension of existential well-being and care burden in spite of controlling other confounding variables. SWB has been reported as one of the psychological factors affecting the care burden. Although other studies have also reported a significant relationship between the care burden and other health dimensions. SWB as the most important health dimension, has an important role in improving the overall health of caregivers. Existential dimension of SWB, in addition to being one of determinant of care burden, acts as a factor in enhancing other aspects of health. Overall, caregiving is a stressful experience; being aware of positive topics such as spirituality, helps therapists provide strategies for caregivers to reduce stress and care burden.

\section{Ethical Considerations}

\section{Compliance with ethical guidelines}

The study obtained its ethical approval from by the Research Ethics Committee of Iran University of Medical Sciences (Code: IR.IUMS.FMD.REC.1397.16). Informed consent was obtained from all the participants. they were free to leave the study whenever they wished, they were also assured about the confidentiality of their information.

\section{Funding}

This study is extracted from the residency thesis of first author School of Behavioral Science and Mental Health (Tehran Institute of Psychiatry), Iran University of Medical Science.

\section{Authors contributions}

Conceptualization: Behnam Shariati; Data collection, data analysis, initial draft preparation: Mahdiyeh Salehi; Discussion: Dr Ruohollah Seddigh; Editing and Review: Vahid Rashedi; Leila Kamalzadeh, Mostafa Almasi-Dooghaee.

\section{Conflicts of interest}

The authors declared no conflict of interest. 


\title{
ارتباط سلامت معنوى با بار مراقبت در مراقبين غيررسمى سالمندان مبتلا به دمانس
}

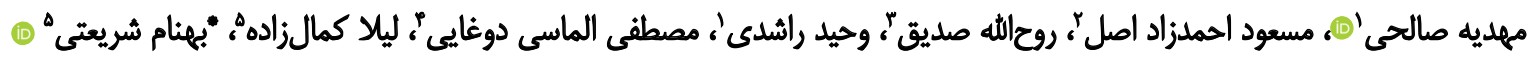

ا. دانشكده علوم رفتارى و سلامت روان (انستيتو روانيزّشكى تهران)، دانشكاه علومئشيكى ايران، تهرانه ايران.

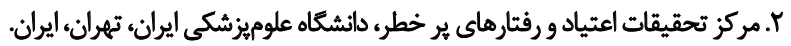

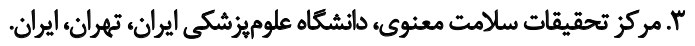

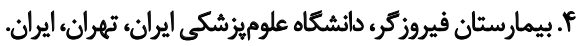

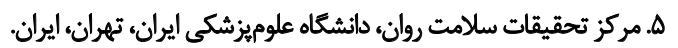

\begin{abstract}
حكيد

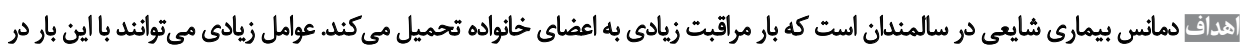

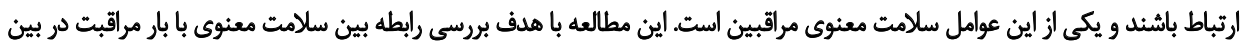

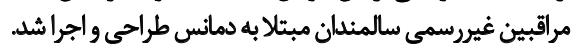

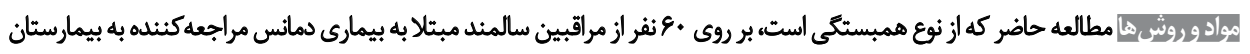

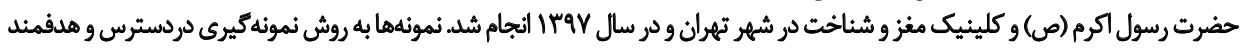

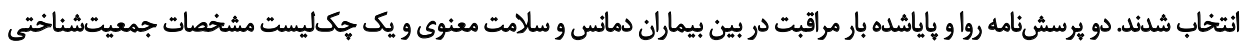

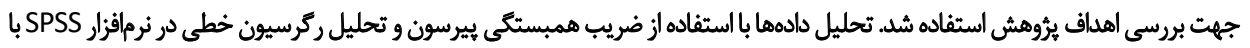
سطع خطاي نوع اول هـ

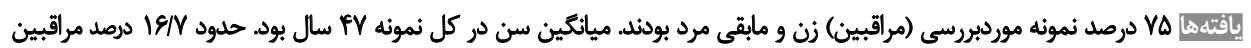

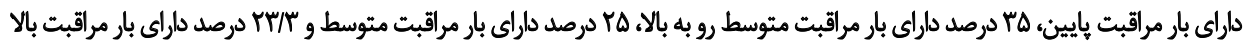

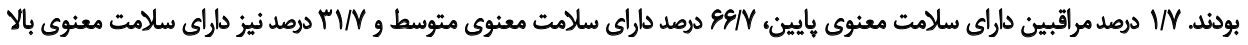

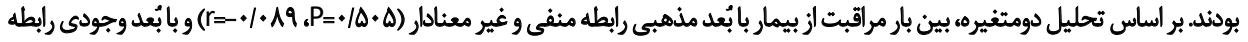

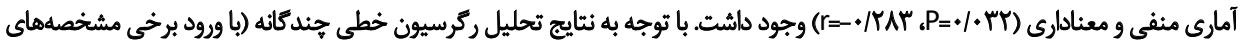

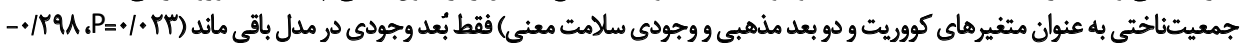

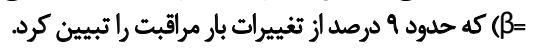

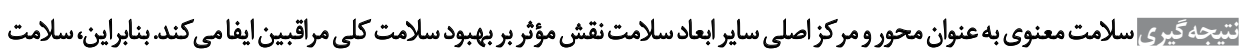

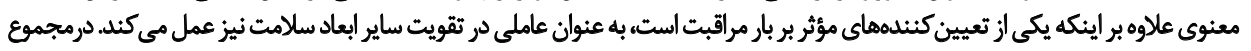

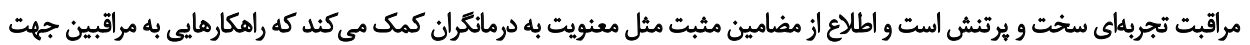
كاهش استرس و بار مراقبت ارئه دهند
\end{abstract}

تاريخ دريافت: م+خرداد

تاريخ يذيرش: 9. بهمن

تاريخ انتشار: با فروردين

\footnotetext{
كليدوارٌهها:

بار مراقبت، دمانس،

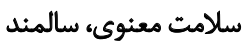

بر FV/D ميليون نفر در سراسر جهان تأثير مي كذارد و سالانه dato

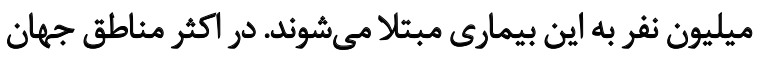

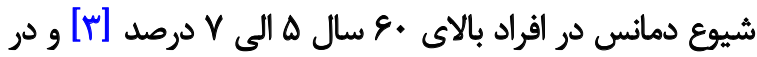

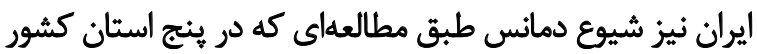

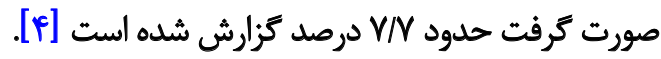
با توجه به شيوع بالاي اين بيمارى در بين سالمندان،

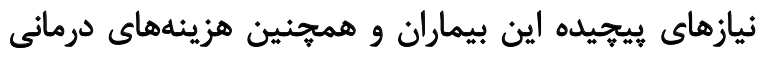

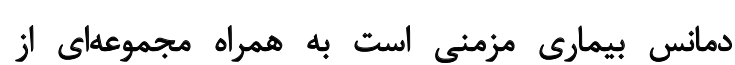

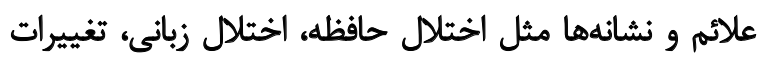

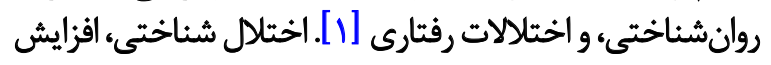

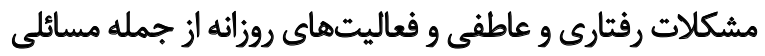

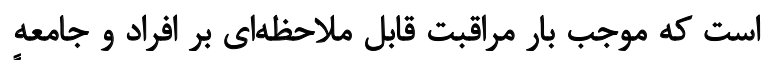

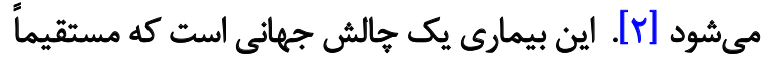


سلامت مذهبى با بار مراقبت رابطه آمارى منفى و معنادارى

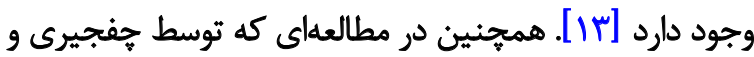

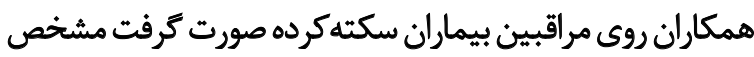

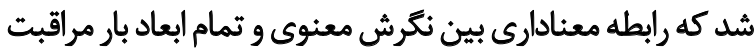

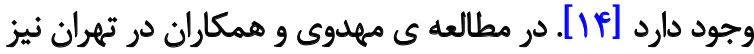

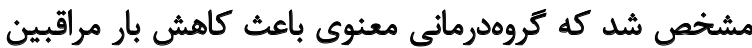

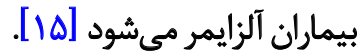

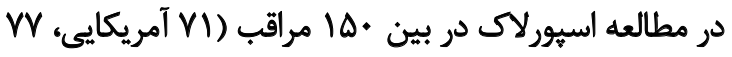

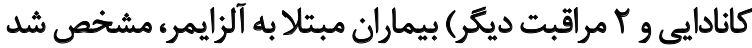

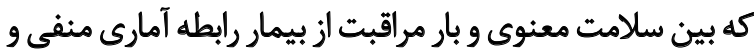

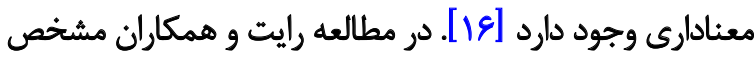

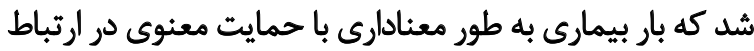

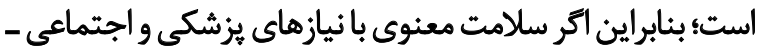

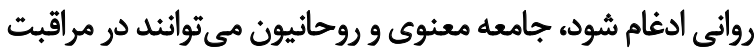
از خانوادههاي بيماران دمانس نقشى ايفا كنئد [IV]

در مطالعه آنوم نيز كه در بين مراقبين (والدين) كودكان مبتلا

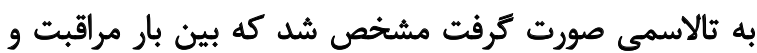

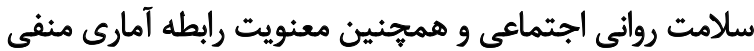

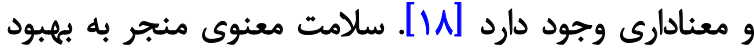

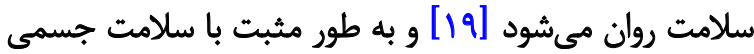

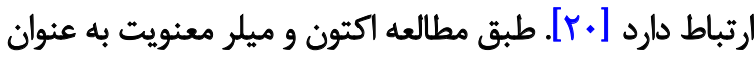

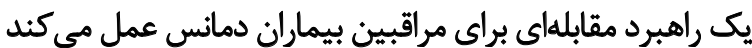

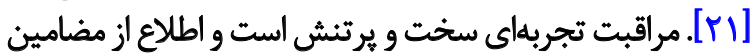

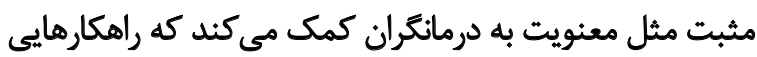

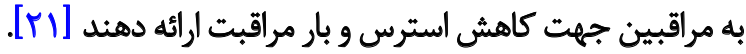

با توجه به اينكه تأثير سلامت معنوى بر بار مراقبت از بيمار

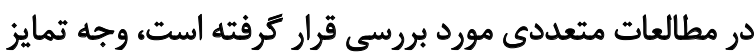

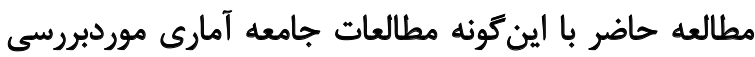

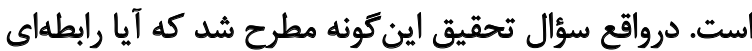

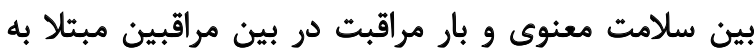

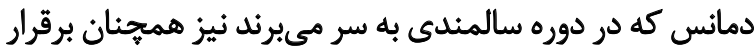

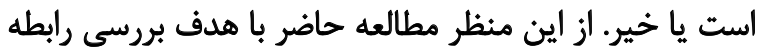

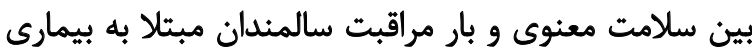
دمانس در شهر تهران طراحي و اجرا مراقيت سالدئ

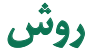

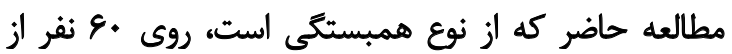

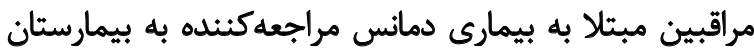

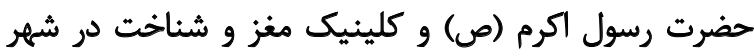

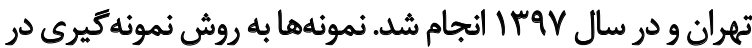

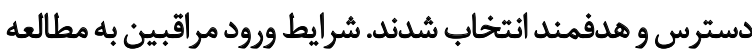

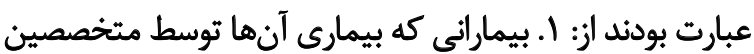

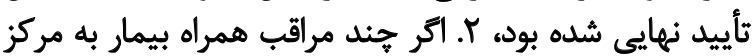

و بار مراقبتى قابل توجهى كه ناشى از طولانى بودن بيمارى و

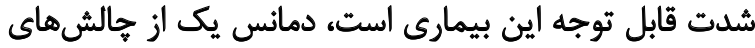

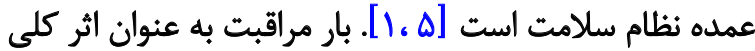

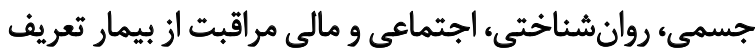
شده است [ع] و ويكى از عوامل ايجادكنينده جالش دالش در رابطه

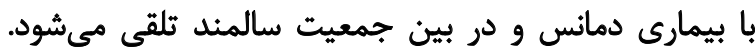

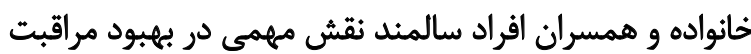

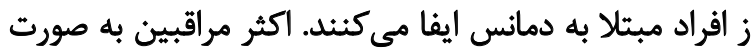

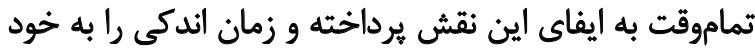

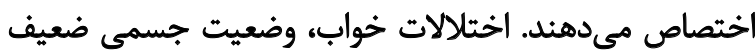

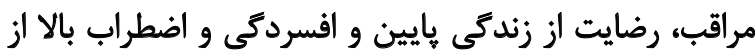

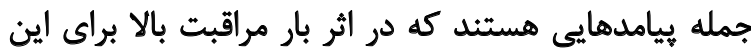

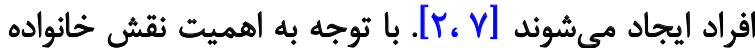

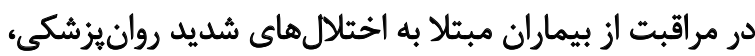

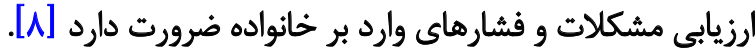

مرور نظاممند بار مراقبت در بين افراد مبتلا به دمانس نيز

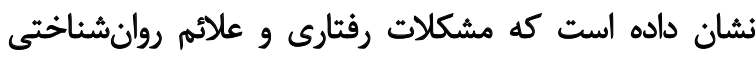

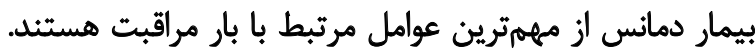

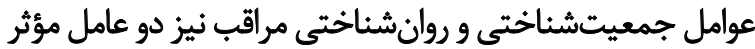

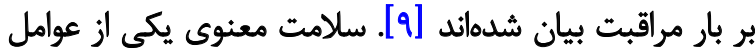

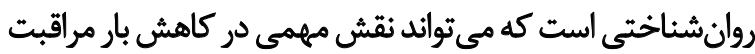

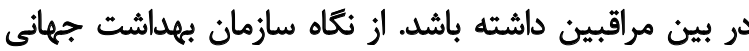

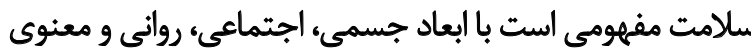
[ [ []] از بين ابعاد يادشده سلامت معنوى مفهومى استى است كه كمتر

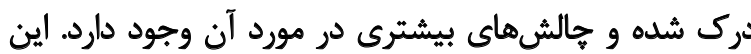

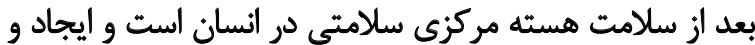

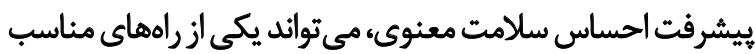
سازكارى با بيمارى باشد [11]. اعتقادات مذهبى و معنوى به عنوان سيرى در برابر استرس،

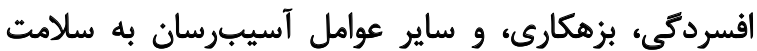

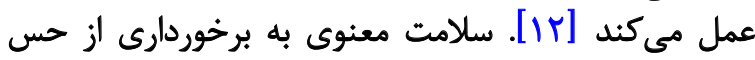

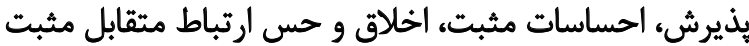

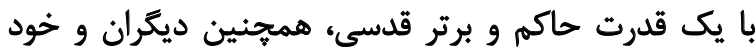

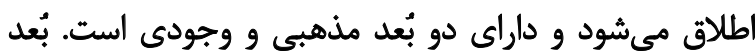

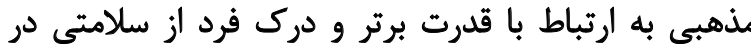

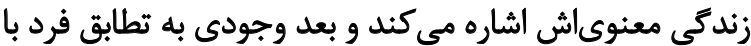

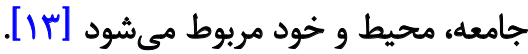

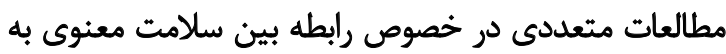

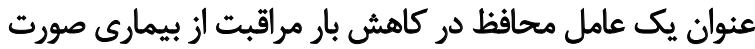

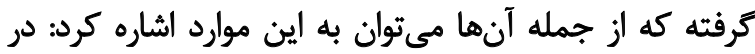

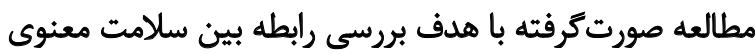

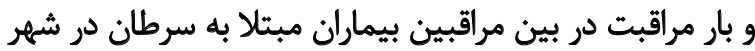

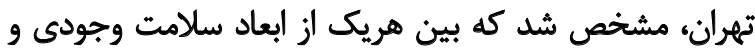




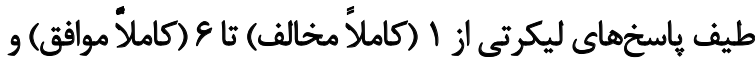

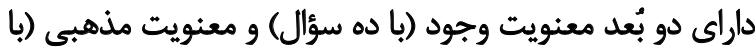

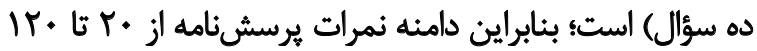

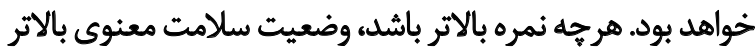

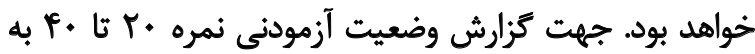

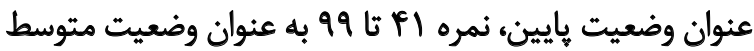

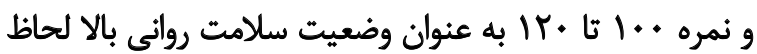

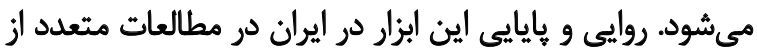

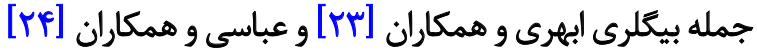

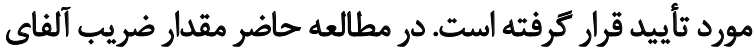

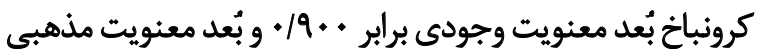

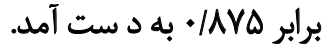

كليه اصول اخلاقي لازم جهت تدوين تا اجراو كَزارش نتايج

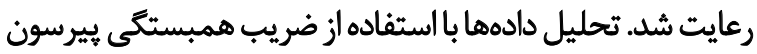

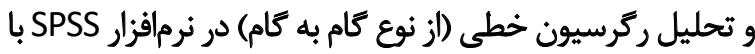

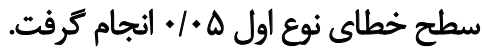

batio

مياكين VD در نمونه مورد بررسى (مراقبين) زن و مابقى مرد بودند.

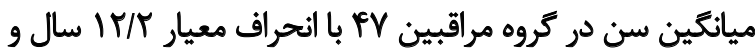

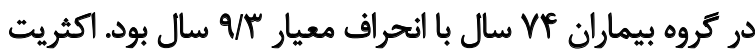

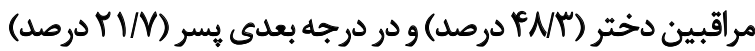

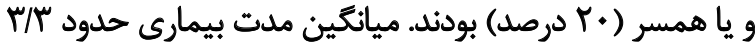

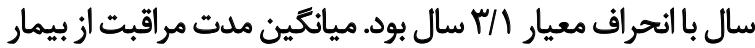

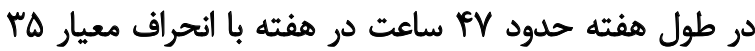

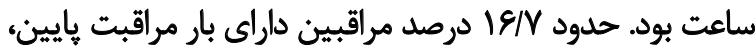

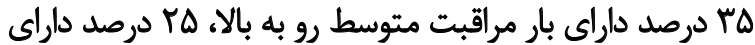

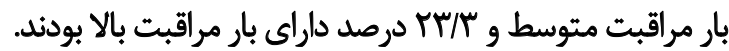

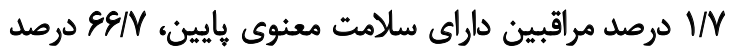

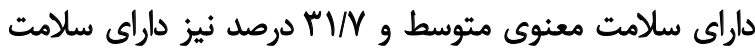

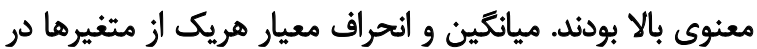

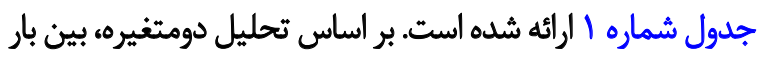

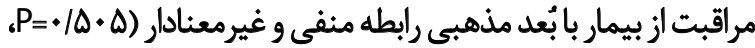

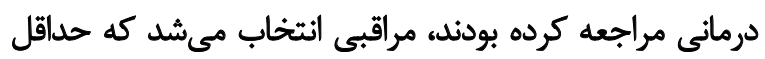

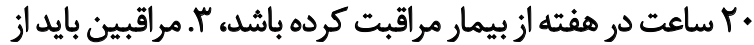

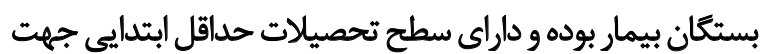

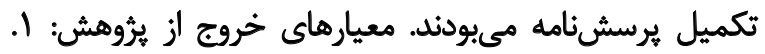

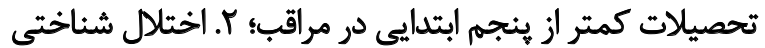

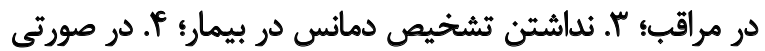

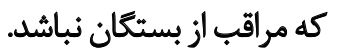

جهت بررسى اهداف تحقيق علاوه بر بررسى ويثگى زهاى

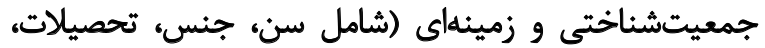

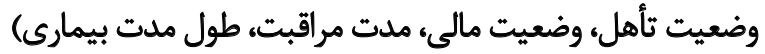

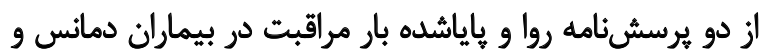

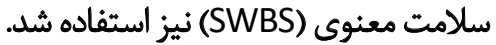

$$
\text { بوسشن بامه بار مراقبث در بيمار ان دمائس' }
$$

اين برسشنامه در ايران توسط عبدالثهيور و همكاران در سال

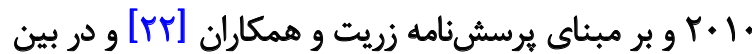

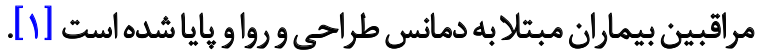

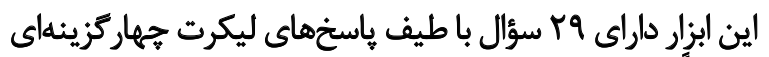

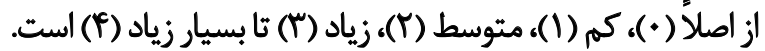

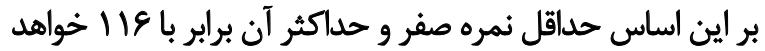

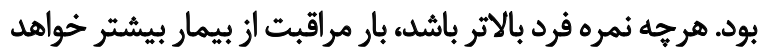

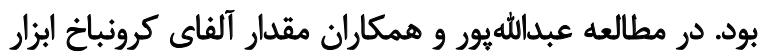

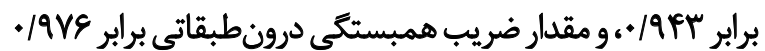

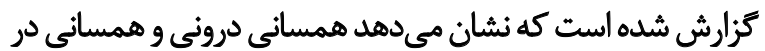

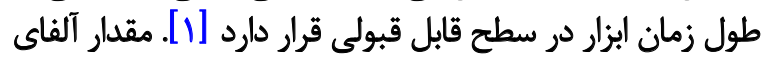

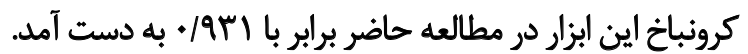

$$
\text { يرسش ثامه سلامث معنوى }
$$

اين مقياس توسط ريموند يالوتزيان و كريك والت اليسون

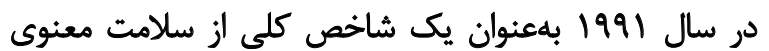

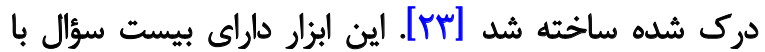

1. Caregiver Burden Questionnaire 2. Spiritual Well-Being Scale

\begin{tabular}{|c|c|}
\hline ميانكين土|نحراف معيار & نمره كل/ ابعاد \\
\hline$P V / T \Delta \pm 1+/ \Delta T$ & بعد وجودى \\
\hline$M T / M \pm 1 \cdot / T T$ & بعد مذهبى \\
\hline$q \cdot / \Delta Y \pm I V \cdot \Delta$ & سلامت معنوى (نمره كل) \\
\hline Pe/IIEYI/RT & تمره كل بار مراقبت \\
\hline
\end{tabular}

جدول ا. توزيع متغير سلامت معنوى در مراقبين

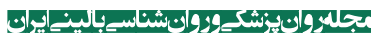


جدول Ү. نتايج تحليل ركرسيون خطى جئدكانه جهت تبيين بار بيمارى بر اساس ويزٔكىهاى جمعيتشناختى و دو بعد مذهبى و وجودى سلامت معنوى

\begin{tabular}{|c|c|c|c|c|c|}
\hline $\mathbf{p}$ & $\mathbf{T}$ & $\boldsymbol{\beta}$ & SE & B & متغير هاى ييشبين \\
\hline $.1+.1$ & 81.9 & - & $M / . r$ & VT/TA & مقدار ثابت \\
\hline r & $-r / M T$ &.$- / r q 1$ & $+/ \pi V$ & $-.19 \pi T$ & بعد وجودى \\
\hline
\end{tabular}

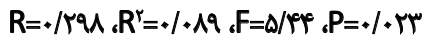

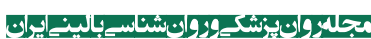

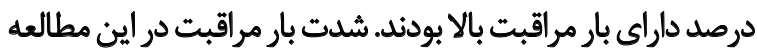

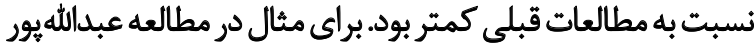

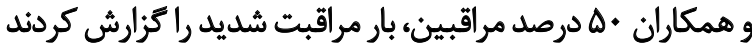

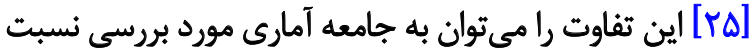

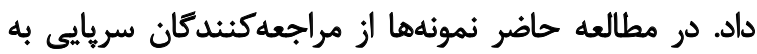

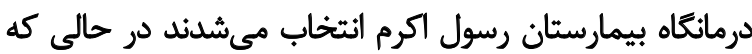

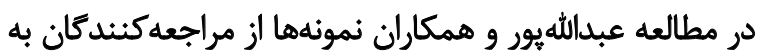

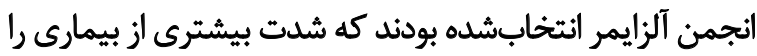

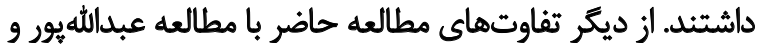
همكاران جامعه آمارى موردبر تفاوسى است.

بر اين اساس از دلايل احتمالى متفاوت بودن وضعيت بار مراقبت

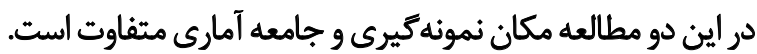

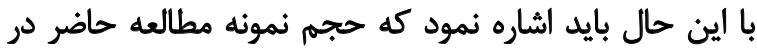

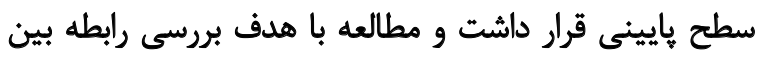

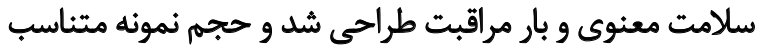

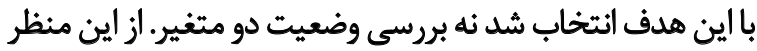

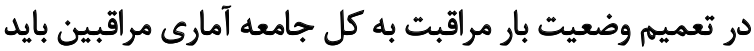

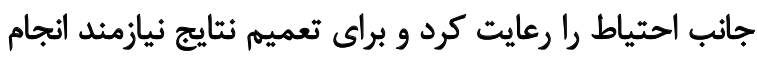
مطالعهاى با حجم نمونه معرف هستيه.

در اين تحقيق مشخص شد كه بين بعد وجودى سلامت

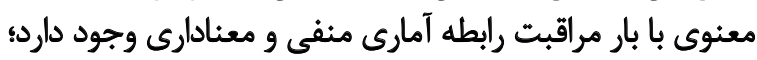

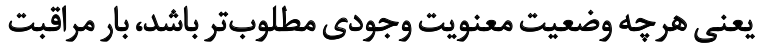

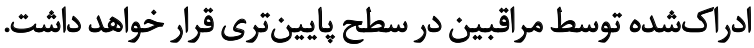

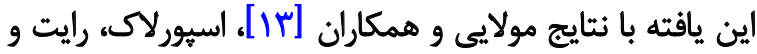

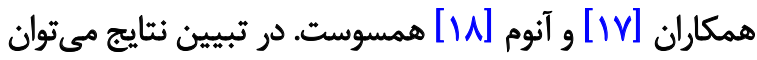

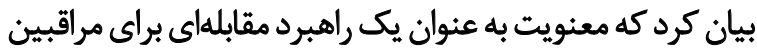

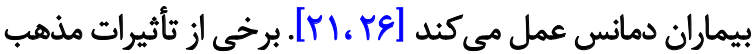

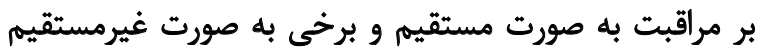

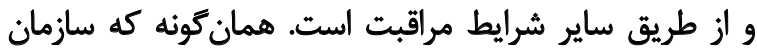

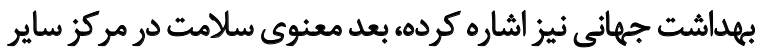

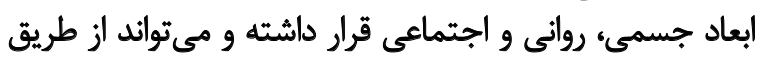

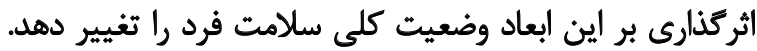

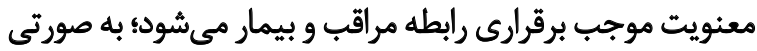

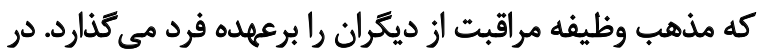

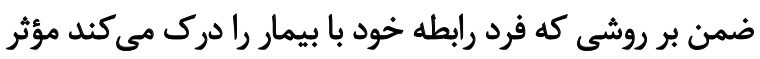

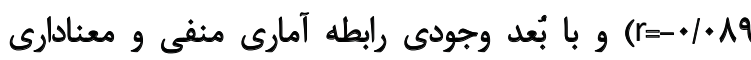

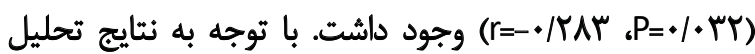

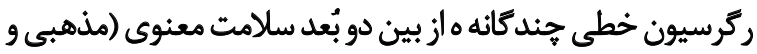

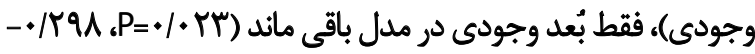

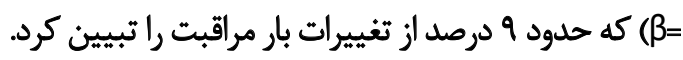

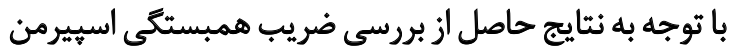

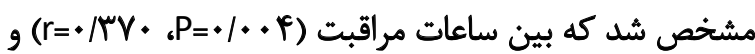

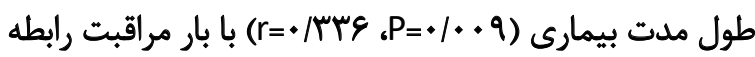

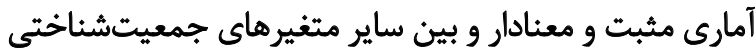

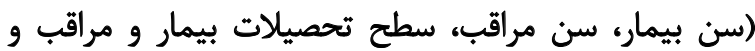

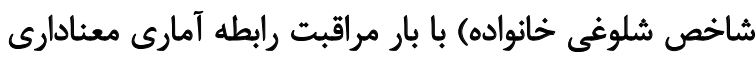

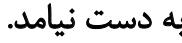

همجينين بين بار بيمارى با نمره كل سلامت معنوى رابطه

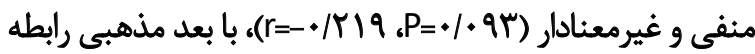

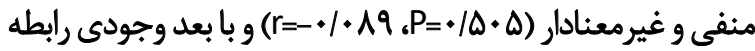

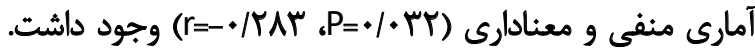

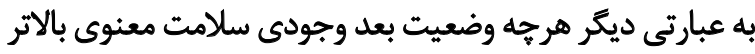

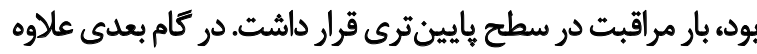

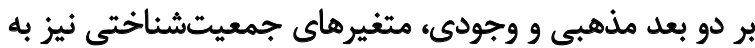

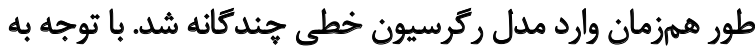

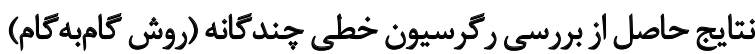

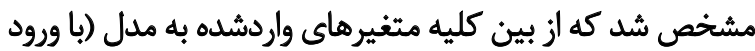

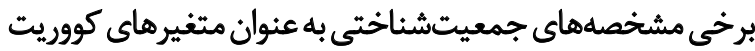

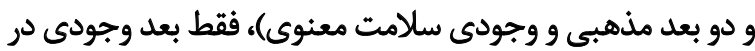

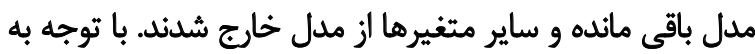

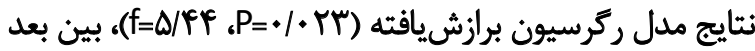

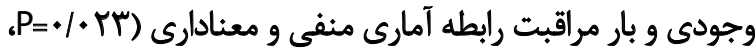

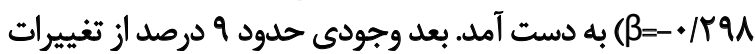

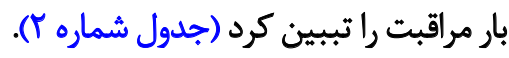

\section{\&y}

مطالعه حاضر با هدف بررسى ارتباط بين سلامت معنوى با بار

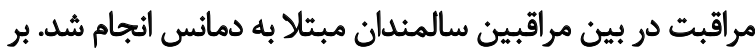

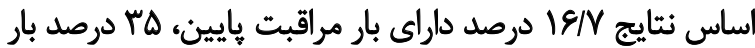

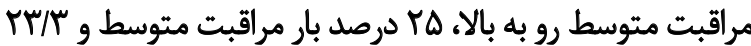


عنوان يك فاكتور همهم مذهب باشند، كوياتر و كاربردى مترند. شايد

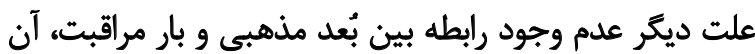

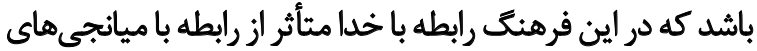

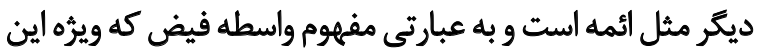

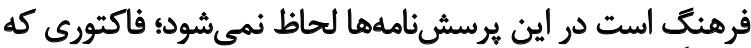

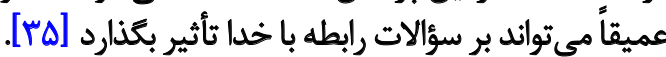

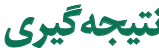

در مطالعه حاضر مشخص شد كه رابطه بين بعد وجودي مندي

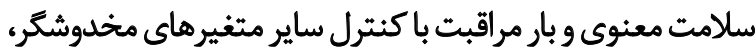

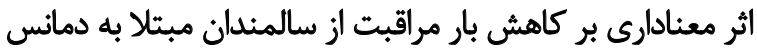

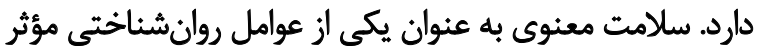

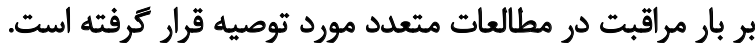

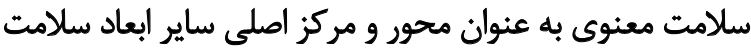

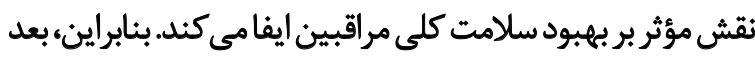

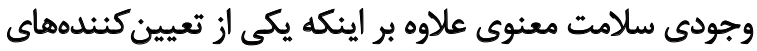

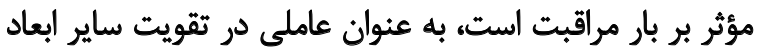

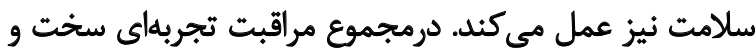

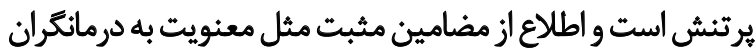

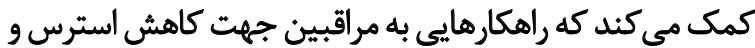

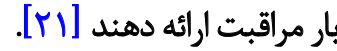

با توجه به شيوع بالاي دمانس، انجام مداخلات كاهنده بار

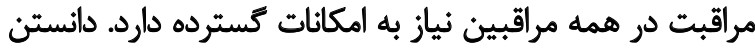

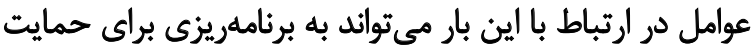

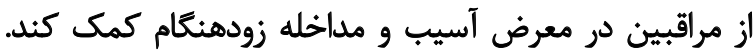

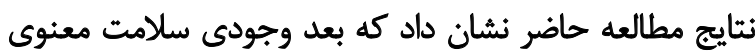

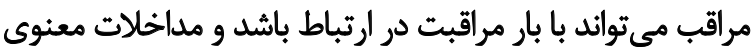

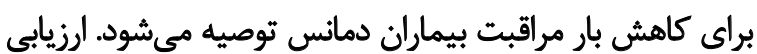

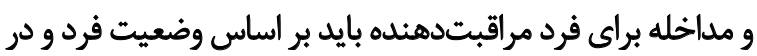
بافتارى صورت گيرد كه بار مراقبت رخ داده است [باست

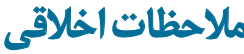

\section{يبروى أز اصول اخحالاق يُوهش}

IR.IUMS.FMD. يروتكل \&ئوهش حاضر (با كد اخلاق

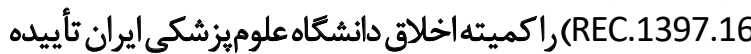

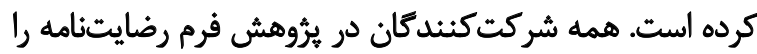

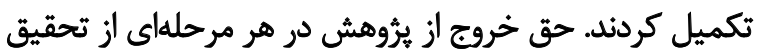

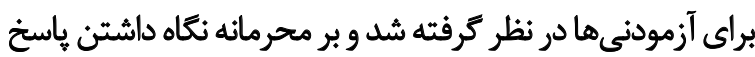
شركت كنند كان همواره تأكيد مى شئد

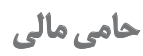

اين ايروهش حاصل ياياننامه دوره دستيارى نويسنده اول

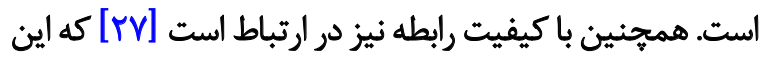

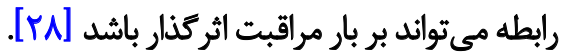

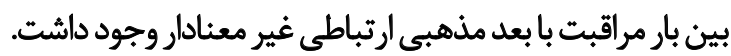

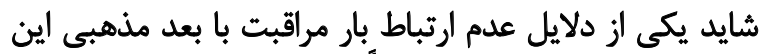

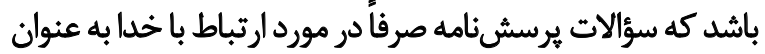

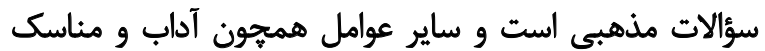

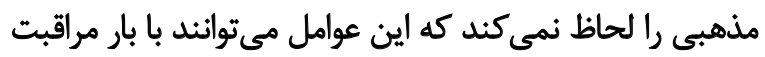

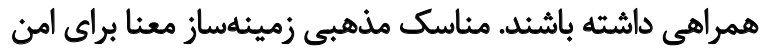

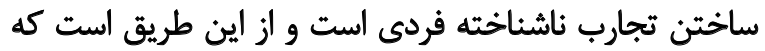

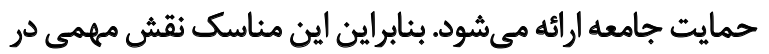

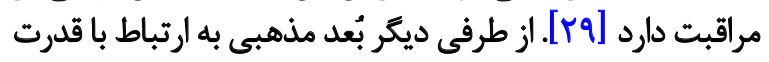

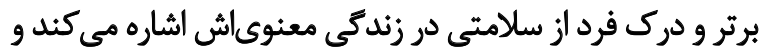

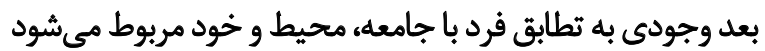

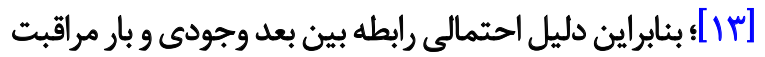

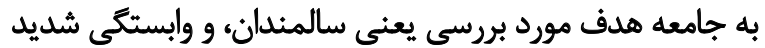

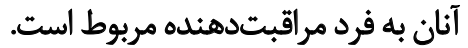

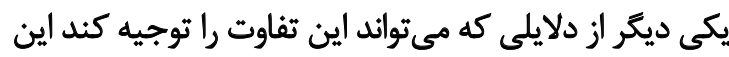

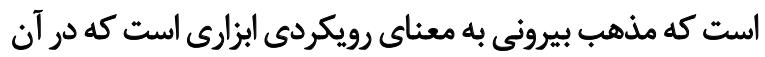

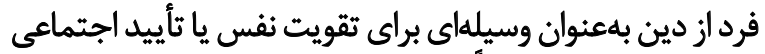

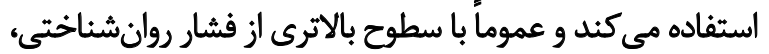

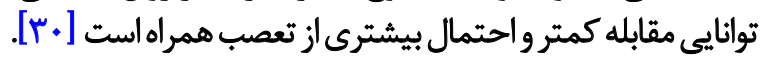

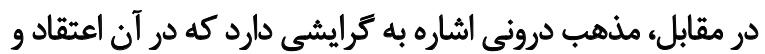

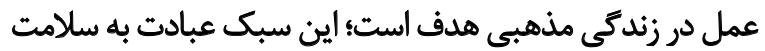

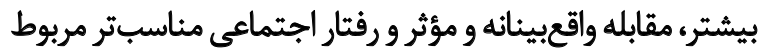

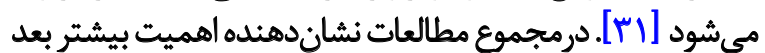

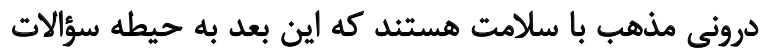

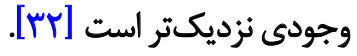

علاوه بر اين، ممكن است افراد در اين مرحله از فشار مراقبت

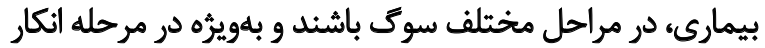

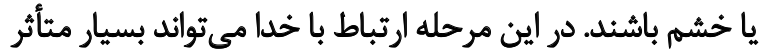

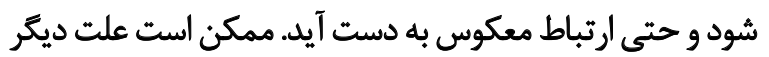

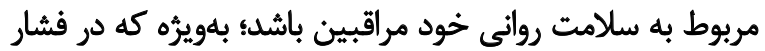

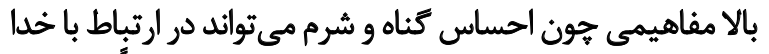

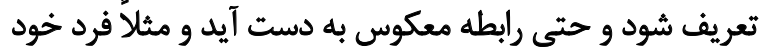

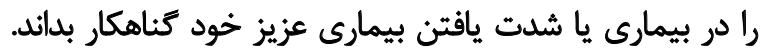

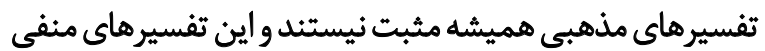

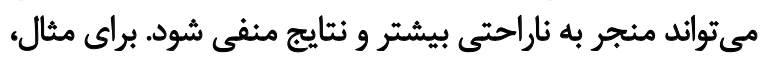

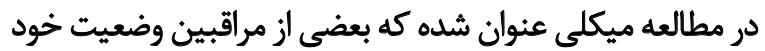

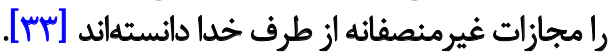

عامل ديكرى كه مي تواند تأثير كذار باشد اين است كه كاهي

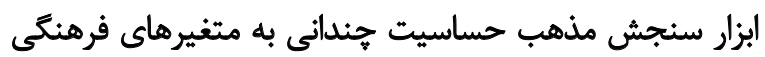

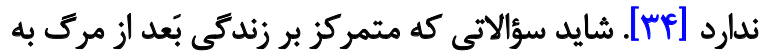


در رشته روانيزشكى در دانشكده علوم رفتارى و سلامت روان

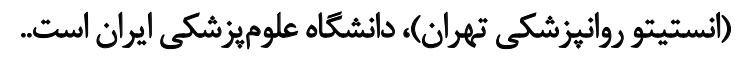

$$
\text { مشاركت نويسند متان }
$$

طراحى يُروهش: بهنام شريعتى. جمعآورى دادهها و تجزيه و

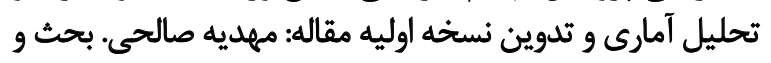

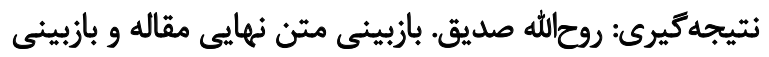

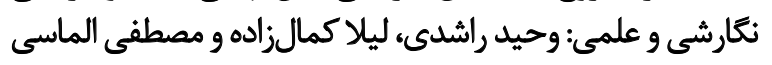
دوغايى.

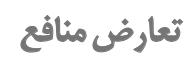
بنابر اظهار نويسندكان، مقاله تعارض منافع ندارد. 


\section{References}

[1] Abdollahpour I, Nedjat S, Noroozian M, Golestan B, Majdzadeh R. Development of a caregiver burden questionnaire for the patients with dementia in Iran. International Journal of Preventive Medicine. 2010; 1(4):233-41. [PMID] [PMCID]

[2] Liu Sh, Li C, Shi Z, Wang X, Zhou Y, Liu Sh, et al. Caregiver burden and prevalence of depression, anxiety and sleep disturbances in A lzheimer's disease caregivers in China. Journal of Clinical Nursing. 2017; 26(9-10):1291-300. [DOI:10.1111/jocn.13601] [PMID]

[3] Prince M, Bryce R, Albanese E, Wimo A, Ribeiro W, Ferri CP. The global prevalence of dementia: A systematic review and metaanalysis. Alzheimer's \& Dementia. 2013; 9(1):63-75.e2. [DOI:10.1016/j.jalz.2012.11.007] [PMID]

[4] Sharifi F, Fakhrzadeh H, Varmaghani M, Arzaghi SM, Alizadeh Khoei M, Farzadfar F, et al. Prevalence of dementia and associated factors among older adults in Iran: National Elderly Health Survey (NEHS). Archives of Iranian Medicine. 2016; 19(12):83844. [PMID]

[5] Svendsboe E, Terum T, Testad I, Aarsland D, Ulstein I, Corbett A, et al. Caregiver burden in family carers of people with dementia with Lewy bodies and Alzheimer's disease. International Journal of Geriatric Psychiatry. 2016; 31(9):1075-83. [DOI:10.1002/ gps.4433] [PMID]

[6] Rezaei Z, Sharifian Sani M, Ostadhashemi L, Ghaedamini Harouni GR. [Quality of life of mothers of children with cancer in Iran (Persian)]. Koomesh. 2018; 20(3):425-31. http:/ / koomeshjournal.semums.ac.ir/article-1-3905-en.html

[7] Kazemi N, Sajjadi H, Vaez-Mahdavi MR, Kamali M, Ghaedamini Harooni GR, Jorjoran Shushtari Z. [Relationship between social capital and mental health among families with disabled members (Persian)]. Journal of Mazandaran University of Medical Sciences. 2017; 27(147):197-206. http://jmums.mazums.ac.ir/article1-9835-en.html

[8] Motaghipour Y, Shams J, Salesian N, Sharifi V, Alaghband Rad J. [Cultural adaptation, validity and reliability of Persian version of experience of caregiving inventory in families of patients with severe mental disorders (Persian)]. Iranian Journal of Psychiatry and Clinical Psychology. 2011; 17(3):226-31. http://ijpcp.iums. ac.ir/article-1-1418-en.html

[9] Chiao CY, Wu HS, Hsiao CY. Caregiver burden for informal caregivers of patients with dementia: A systematic review. International Nursing Review. 2015; 62(3):340-50. [DOI:10.1111/ inr.12194] [PMID]

[10] Ghaedamini Harouni GR, Sajjadi H, Rafiey H, Mirabzadeh A, Vaez-Mahdavi MR, Mohaqeqi Kamal SH. Current status of health index in Tehran: A multidimensional approach. Medical Journal of the Islamic Republic of Iran. 2017; 31(1):29. [DOI:10.18869/ mjiri.31.29] [PMID] [PMCID]

[11] Ross L, Giske T, van Leeuwen R, Baldacchino D, McSherry W, Narayanasamy A, et al. Factors contributing to student nurses' / midwives' perceived competency in spiritual care. Nurse Education Today. 2016; 36:445-51. [DOI:10.1016/j.nedt.2015.10.005] [PMID]

[12] Ghobari Bonab B. [Religious beliefs and its effects on mental health (Persian)]. Iranian Journal of Psychiatry and Clinical
Psychology. 1995; 1(4):48-54. http://ijpcp.iums.ac.ir/article1-1666-en.html

[13] Mollaei F, Borhani F, Abbaszadeh A, Khabazkhoob M. [Correlation between spiritual well-being and burden of care in family caregivers of cancer patients (Persian)]. Journal of Hayat. 2019; 24(4):296-309. http:/ / hayat.tums.ac.ir/article-1-2583-en. html

[14] Torabi Chafjiri R, Navabi N, Shamsalinia A, Ghaffari F. The relationship between the spiritual attitude of the family caregivers of older patients with stroke and their burden. Clinical Interventions in Aging. 2017; 12:453-8. [DOI:10.2147/CIA.S121285] [PMID] [PMCID]

[15] Mahdavi B, Fallahi-Khoshknab M, Mohammadi F, Hosseini MA, Haghi M. Effects of spiritual group therapy on caregiver strain in home caregivers of the elderly with Alzheimer's disease. Archives of Psychiatric Nursing. 2017; 31(3):269-73. [DOI:10.1016/j.apnu.2016.12.003] [PMID]

[16] Spurlock WR. Spiritual well-being and caregiver burden in Alzheimer's caregivers. Geriatric Nursing. 2005; 26(3):154-61. [DOI:10.1016/j.gerinurse.2005.03.006] [PMID]

[17] Wright SD, Pratt CC, Schmall VL. Spiritual support for caregivers of dementia patients. Journal of Religion and Health. 1985; 24(1):31-8. [DOI:10.1007/ BF01533257] [PMID]

[18] Anum J, Dasti R. Caregiver burden, spirituality, and psychological well-being of parents having children with thalassemia. Journal of Religion and Health. 2016;55(3):941-55. [DOI:10.1007/ s10943-015-0127-1] [PMID]

[19] Hilton JM, Child SL. Spirituality and the successful aging of older Latinos. Counseling and Values. 2014; 59(1):17-34. [DOI:10.1002/j.2161-007X.2014.00039.x]

[20] Hematti S, Baradaran-Ghahfarokhi M, Khajooei-Fard R, Mohammadi-Bertiani Z. Spiritual well-being for increasing life expectancy in palliative radiotherapy patients: A questionnairebased study. Journal of Religion and Health. 2015; 54(5):1563-72. [DOI:10.1007/s10943-014-9872-9] [PMID]

[21] Acton GJ, Miller EW. Spirituality in caregivers of family members with dementia. Journal of Holistic Nursing. 2003; 21(2):11730. [DOI:10.1177/0898010103021002003] [PMID]

[22] Zarit SH, Todd PA, Zarit JM. Subjective burden of husbands and wives as caregivers: A longitudinal study. The Gerontologist. 1986; 26(3):260-6. [DOI:10.1093/geront/26.3.260] [PMID]

[23] Biglari Abhari M, Fisher JW, Kheiltash A, Nojomi M. Validation of the Persian version of spiritual well-being questionnaires. Iranian Journal of Medical Sciences. 2018; 43(3):276-85. [DOI:10.30476/IJMS.2018.40539]

[24] Abbasi M, Farahani-Nia M, Mehrdad N, Givari A, Haghani H. Nursing students' spiritual well-being, spirituality and spiritual care. Iranian Journal of Nursing and Midwifery Research. 2014; 19(3):242-7. [PMID] [PMCID]

[25] Abdollahpour I, Noroozian M, Nedjat S, Majdzadeh R. Caregiver burden and its determinants among the family members of patients with dementia in Iran. International Journal of Preventive Medicine. 2012; 3(8):544-51. [PMID] [PMCID]

[26] Guberman N, Maheu P, Maillé C. Women as family caregivers: Why do they care? The Gerontologist. 1992; 32(5):607-17. [DOI:10.1093/geront/32.5.607] [PMID] 
[27] Dudley MG, Kosinski Jr FA. Religiosity and marital satisfaction: A research note. Review of Religious Research. 1990; 32(1):78-86. [DOI:10.2307/3511329]

[28] Draper BM, Poulos RG, Poulos CJ, Ehrlich F. Risk factors for stress in elderly caregivers. International Journal of Geriatric Psychiatry. 1996; 11(3):227-31. [DOI:10.1002/(SICI)10991166(199603)11:3<227::AID-GPS311>3.0.CO;2-W

[29] Sankar A. Ritual and dying: A cultural analysis of social support for caregivers. The Gerontologist. 1991; 31(1):43-50. [DOI:10.1093/geront/31.1.43] [PMID]

[30] Smith TB, McCullough ME, Poll J. Religiousness and depression: Evidence for a main effect and the moderating influence of stressful life events. Psychological Bulletin. 2003; 129(4):614-36. [DOI:10.1037/0033-2909.129.4.614] [PMID]

[31] Batson CD, Schoenrade P, Ventis WL. Religion and the individual: A social-psychological perspective. Oxford: Oxford University Press; 1993. https://psycnet.apa.org/record/1993-98302-000

[32] Tsuang MT, Simpson JC, Koenen KC, Kremen WS, Lyons MJ. Spiritual well-being and health. The Journal of Nervous and Mental Disease. 2007; 195(8):673-80. [DOI:10.1097/ NMD.0b013e31811f4062] [PMID]

[33] Mickley JR, Pargament KI, Brant CR, Hipp KM. God and the search for meaning among hospice caregivers. The Hospice Journal. 1998; 13(4):1-17. [DOI:10.1080/0742-969X.1998.11882904] [PMID]

[34] Chatters LM, Taylor RJ, Lincoln KD, Schroepfer T. Patterns of informal support from family and church members among African Americans. Journal of Black Studies. 2002; 33(1):66-85. [DOI:1 0.1177/002193470203300104]

[35] Amiri P, Abbasi M, Gharibzadeh S, Asghari Jafarabadi M, Hamzavi Zarghani N, Azizi F. [Designation and psychometric assessment of a comprehensive spiritual health questionnaire for Iranian populations (Persian)]. Medical Ethics Journal. 2015; 9(30):25-56. http://ojs2.sbmu.ac.ir/en-me/article/ view/10066 
This Page Intentionally Left Blank 